\title{
Changes on fecal microbiota in rats exposed to permethrin during postnatal development
}

\author{
Cinzia Nasuti $^{1}$ (D) Maria Magdalena Coman ${ }^{2} \cdot$ Robert A. Olek $^{3} \cdot$ Dennis Fiorini $^{4}$. \\ Maria Cristina Verdenelli ${ }^{2,5} \cdot$ Cinzia Cecchini $^{2,5} \cdot$ Stefania Silvi $^{2}$ - Donatella Fedeli ${ }^{1}$. \\ Rosita Gabbianelli ${ }^{1}$
}

Received: 16 October 2015 / Accepted: 14 February 2016

(C) Springer-Verlag Berlin Heidelberg 2016

\begin{abstract}
Alteration of the gut microbiota through diet and environmental contaminants may disturb the mammalian digestive system, leading to various diseases. Because most exposure to environmentally pyrethroid pesticides such as permethrin (PERM) occurs through the diet, the commensal gut microbiota is likely to be exposed to PERM. The study aimed at evaluating the effect of low-dose exposure to PERM in early life on the composition of fecal microbiota in rats. Over a 4-month follow-up period, fecal microbiota and short-chain fatty acids were measured in order to identify possible differences between PERM-treated rats and controls. Further in vitro antimicrobial experiments were conducted to establish the antibacterial activity of PERM against different strains to obtain Minimal Inhibitory Concentrations. The main finding focused on the reduced abundance of BacteroidesPrevotella-Porphyromonas species, increased
\end{abstract}

Responsible editor: Philippe Garrigues

Cinzia Nasuti and Maria Magdalena Coman contributed equally to this work.

Cinzia Nasuti

cinzia.nasuti@unicam.it

1 School of Pharmacy, Via Madonna delle Carceri, University of Camerino, 62032 Camerino, Italy

2 School of Biosciences and Veterinary Medicine, University of Camerino, 62032 Camerino, Italy

3 Department of Bioenergetics and Nutrition, Gdansk University of Physical Education and Sport, Kazimierza Górskiego 1, 80-336 Gdańsk, Poland

4 School of Science and Technology-Chemistry Division, University of Camerino, 62032 Camerino, Italy

5 Synbiotec S.r.l., Camerino, Italy
Enterobacteriaceae and Lactobacillus in PERM-treated rats compared to controls. Changes of acetic and propionic acid levels were registered in PERM-treated group. From in vitro studies, PERM showed higher antibacterial activity against beneficial bacteria such as Bifidobacterium and Lactobacillus paracasei, while to inhibit potential pathogens as Staphylococcus aureus and Escherichia coli PERM concentration needed to be increased. In summary, exposure to PERM could affect the fecal microbiota and could be a crucial factor contributing to the development of diseases.

Keywords Permethrin · Postnatal exposure · Gutmicrobiota · Short-chain fatty acids $\cdot$ Environmental contaminants

\section{Introduction}

Due to an increasing concern about adverse health consequences, organophosphate, carbamate, and organochlorine are being phased out leading to escalating use of an alternative class of pesticides, the pyrethroids. Pyrethroids are now the most commonly used pesticides for residential pest control, public health purposes, and for agriculture with biomonitoring studies confirming widespread exposure to one or more pyrethroids (Williams et al. 2008; Morgan 2012; Barr et al. 2010; Heudorf and Angerer 2001; Roberts et al. 2012). One of the main routes of pyrethroid exposure involves dietary residues through food contamination (Barr et al. 2010; Heudorf and Angerer 2001).

Although pyrethroid pesticides are often considered a "safer" choice because they are generally not as acutely toxic as organophosphates (Casida and Durkin 2013), our studies on adult and adolescent rats indicated that oral long-term exposure to permethrin (PERM), one of the most representative pyrethroid compound, may not be benign (Nasuti et al. 2003; Gabbianelli et al. 2004; Nasuti et al. 2008). In particular, 
oxidative damage to lymphocyte and erythrocyte was observed at doses of $150 \mathrm{mg} / \mathrm{kg}$. However, a worst effect of PERM was observed when it was administered to rat pups during the suckling period from postnatal day (PND) 6 to PND 21 at doses of $34 \mathrm{mg} / \mathrm{kg}$ close to the No Observed Adverse Effects Levels (NOAEL) for PERM (25 mg/kg). Daily oral dose of PERM for 15 days induced dopamine and glutathione depletion together with lipid peroxidation in the basal ganglia of brain (Nasuti et al. 2007).

In mammals, the postnatal period is characterized by the progressive colonization of the gut ecosystem by bacteria acquired during the delivery process, from the mother's skin and milk and other environmental and oral sources (Morelli 2008). Subsequently, the presence of pesticide residues in food of young animals could induce variations in the gut microbiota that is essential for the development and maturation of gastrointestinal tract and enteric immune system, the maintenance of barrier function and the provision of essential nutrients. Furthermore, alterations in the colonization process predispose and increase the risk to disease later in life.

Given that weaning is a critical period for bacterial profile changes, we investigated, here, the impact of daily, oral exposure to low-dose PERM (34 mg/kg), from PND 6 to PND 21, on the fecal microbiota by using an in vivo model.

The changes over time in the populations of fecal microflora were examined at four different time points: PND 21 (weaning), PND 51 (adolescent age), PND 81 and PND 141 (adulthood). In parallel, control rats were gavaged daily with vehicle in order to establish possible differences between control and PERM-treated rats. In the same samples, we measured the fecal levels of short-chain fatty acids (SCFAs), which are the principle nutrient substrates of the intestinal epithelial cells and are themselves produced by commensal anaerobic bacteria via carbohydrate fermentation.

In vitro experiments were performed to establish the antibacterial activity of PERM against 16 different strains (Grampositives, Gram-negatives and yeasts) in order to obtain Minimal Inhibitory Concentrations (MICs). Moreover, we examined the effect of PERM on the relative levels of different bacterial groups present in the human fecal microbiota using a semi-continuous fermentor mimicking ileostomy fluid.

\section{Materials and methods}

\section{In vitro studies}

\section{MICs of PERM}

The in vitro experiments were performed using 16 different strains: Gram-positives, Gram-negatives and yeasts. The strains, their origins and their cultural and growth conditions were described in the Table 1.
For each tested strains MIC was determined using a broth microdilution method with a 96-well microtiter plate (Clinical and Laboratory Standards Institute 2007). One series of 2-fold dilutions of PERM in dimethyl sulfoxide and distilled water (DMSO: $\mathrm{H}_{2} \mathrm{O}$ ranging from 3.2 to $0.00625 \mathrm{mg} / \mathrm{mL}$ ) for each microbial strain was prepared using the mean mentioned in the supplementary material. Each series was inoculated with $0.8 \mu \mathrm{L}$ of each microbial strain $\left(1.5 \times 10^{8}\right.$ cells $\left./ \mathrm{ml}\right)$. MIC determination, as the lowest concentration of PERM that inhibited the visible growth of the microorganisms after overnight $/ 24 / 48 \mathrm{~h}$ of incubation at $37^{\circ} \mathrm{C}$, was carried out evaluating the microbial growth in the wells using a stereomicroscope (GSZ2, Ascania, Germany).

\section{Fermentation study}

The medium used in this semi-continuous fermenter system was complex and designed to mimic either ileostomy fluid or ileal chime. The composition and the addition of fecal fluid and primary bile acids in the medium were those reported by Zampa et al. (2004). Two experiments were performed (with three replicates for each): (i) a fermentation with semicontinuous culture with the addition of glucose in the medium (control test); (ii) a fermentation with semi-continuous culture with addition of $6 \mathrm{~mL}$ of PERM (1.28 $\mathrm{mg}$ in corn oil) in the medium each day. Each fermentation was run for 5 days.

A pool of freshly voided fecal samples from healthy volunteers with no history of gastrointestinal disease, both before and during the sampling period, and not undergoing antibiotic therapy one month prior to sampling, was used as inoculum. The procedures used in preparing the inoculum were reported in Zampa et al. (2004).

The viable bacteria count (aerobes and anaerobes) was estimated by spreading tenfold dilutions of the original sample into selective agars (Silvi et al. 1996; Zampa et al. 2004). The bacteriological analysis was performed at different times of fermentation: immediately after inoculation $(0 \mathrm{~h}), 24,48,72$, and $96 \mathrm{~h}$. The agar plates were spread in duplicate with $50 \mu \mathrm{L}$ of diluted sample and then incubated in aerobic and anaerobic conditions at $37^{\circ} \mathrm{C}$ for $24-72 \mathrm{~h}$.

\section{In vivo studies}

\section{Materials}

Technical grade (75: 25, trans: cis; $94 \%$ purity) 3phenoxybenzyl-(1R,S)-cis,trans-3-(2,2-dichlorovinyl)-2,2dimethylcyclopropanecarboxyl-ate, PERM (NRDC 143) were generously donated by Dr. A. Stefanini of ACTIVA (Milan, Italy). Corn oil was obtained from Sigma (Milan, Italy). 
Table 1 Target strains used for MIC determination, their origin, cultural, and growth conditions

\begin{tabular}{|c|c|c|c|c|}
\hline & Strain & Strain code & Origin & Cultural and growth conditions \\
\hline \multirow[t]{12}{*}{ Gram-positives } & Bacillus subtilis & ATCC 6633 & Culture collection & $\mathrm{TSB}, 24 \mathrm{~h}$ at $37^{\circ} \mathrm{C}$, aerobiosis \\
\hline & Blautia producta & DSM 2950 & Culture collection & MRS enriched with Cysteine, $24-48 \mathrm{~h}$ at $37{ }^{\circ} \mathrm{C}$, anaerobiosis \\
\hline & Bifidobacterium spp. & A1 & Human intestinal tract & MRS enriched with Cysteine, $24-48 \mathrm{~h}$ at $37^{\circ} \mathrm{C}$, anaerobiosis \\
\hline & Bifidobacterium spp. & $\mathrm{A} 2$ & Human intestinal tract & MRS enriched with Cysteine, $24-48 \mathrm{~h}$ at $37^{\circ} \mathrm{C}$, anaerobiosis \\
\hline & Bifidobacterium spp. & B1 & Human intestinal tract & MRS enriched with Cysteine, $24-48 \mathrm{~h}$ at $37^{\circ} \mathrm{C}$, anaerobiosis \\
\hline & Bifidobacterium spp. & $\mathrm{F} 1$ & Human intestinal tract & MRS enriched with Cysteine, $24-48 \mathrm{~h}$ at $37^{\circ} \mathrm{C}$, anaerobiosis \\
\hline & Enterococcus faecalis & ATCC 29218 & Culture collection & TSB, $24 \mathrm{~h}$ at $37^{\circ} \mathrm{C}$, aerobiosis \\
\hline & Lactobacillus paracasei & IMC $502^{\circledR}$ & Human intestinal tract & MRS with Vancomycin, $48 \mathrm{~h}$ at $37^{\circ} \mathrm{C}$, aerobiosis \\
\hline & Lactobacillus plantarum & $\operatorname{IMC} 512^{\circledR}$ & Human intestinal tract & MRS with Vancomycin, $48 \mathrm{~h}$ at $37^{\circ} \mathrm{C}$, aerobiosis \\
\hline & Lactobacillus rhamnosus & $\operatorname{IMC} 501^{\circledR}$ & Human intestinal tract & MRS with Vancomycin, $48 \mathrm{~h}$ at $37^{\circ} \mathrm{C}$, aerobiosis \\
\hline & Staphylococcus aureus & ATCC 25923 & Culture collection & $\mathrm{TSB}, 24 \mathrm{~h}$ at $37^{\circ} \mathrm{C}$, aerobiosis \\
\hline & Streptococcus mutans & ATCC 20523 & Culture collection & $\mathrm{TSB}, 24 \mathrm{~h}$ at $37^{\circ} \mathrm{C}$, aerobiosis \\
\hline \multirow[t]{2}{*}{ Gram-negatives } & Escherichia coli & ATCC 13706 & Culture collection & $\mathrm{TSB}, 24 \mathrm{~h}$ at $37^{\circ} \mathrm{C}$, aerobiosis \\
\hline & Pseudomonas aeruginosa & DSM 1117 & Culture collection & $\mathrm{TSB}, 24 \mathrm{~h}$ at $37^{\circ} \mathrm{C}$, aerobiosis \\
\hline \multirow[t]{2}{*}{ Yeasts } & Candida albicans & ATCC 14053 & Culture collection & $\mathrm{SAB}, 24 \mathrm{~h}$ at $37^{\circ} \mathrm{C}$, aerobiosis \\
\hline & Candida albicans & ATCC 10261 & Culture collection & $\mathrm{SAB}, 24 \mathrm{~h}$ at $37^{\circ} \mathrm{C}$, aerobiosis \\
\hline
\end{tabular}

\section{Animals and treatment}

Male and female Wistar rats aged about 90 days weighing 250$270 \mathrm{~g}$ were obtained from Charles River (Calco, LC, Italy). The animals were housed in plastic (Makrolon) cages (five rats/ cage) in a temperature controlled room $\left(21 \pm 5^{\circ} \mathrm{C}\right)$ and maintained on a standard GLP diet (4RF21, Mucedola S.r.l., Settimo Milanese, Milan, Italy) with water ad libitum. The composition of the standard diet was: raw proteins $167.4 \mathrm{~g} / \mathrm{kg}$, raw fats $20.0 \mathrm{~g} / \mathrm{kg}$, raw fibers $71.7 \mathrm{~g} / \mathrm{kg}$, raw ashes $63.3 \mathrm{~g} / \mathrm{kg}$.

The light/dark cycle was from 7 a.m. to 7 p.m. Male rat pups born in our laboratory from primiparous dams were used in the study. PERM-treated rats were treated daily by gavage with a PERM solution (34 mg/4 mL/kg body weight) from PND 6 to PND 21, whereas control rats were treated with the vehicle (corn oil $4 \mathrm{~mL} / \mathrm{kg}$ body weight) on a similar schedule as previously described (Nasuti et al. 2013). The PERM solution was obtained by dissolving PERM in corn oil. Both control and PERM-treated groups were given free access to standard rat diet.

All procedures were carried out in accordance with the European guidelines (Directive 2010/63/EU) for the care and use of laboratory animals.

\section{Experimental design}

At the end of the treatment (PND 21) with PERM or vehicle, fecal pellets were freshly collected from six PERM-treated and six control rats; pellets were stored at $-80{ }^{\circ} \mathrm{C}$ until analysis of the microbiota by RT-PCR. Moreover, at PND 51, 81, and 141, fecal pellets were again collected and stored at
$-80{ }^{\circ} \mathrm{C}$ until analysis to quantify selected bacterial strains and SCFAs.

For collection of feces, rats were held in metabolic cages. After $24 \mathrm{~h}$, fecal output and food intake were measured.

\section{Bacterial quantification by RT-PCR from fecal sample}

A Real-Time quantitative PCR (QPCR) procedure was used for the quantification of the main bacterial groups of rats' intestinal microbiota by using specific primers, chosen from literature (Rinttila et al. 2004; Fang and Hedin 2003; Byun et al. 2004; Langendijk et al. 1995; Bartosch et al., 2004) as shown in the Table 2. From each fecal sample the DNA was extracted using Stool DNA isolation kit (Norgen, Thorold, Canada). SYBR Green Real-Time PCR amplification was performed in triplicate using an iCycler iQ Real-Time Detection System (Stratagene) associated with MXP Software using the conditions reported in the supplementary material. For quantification of the above-mentioned target groups of bacteria, standard curves, previously generated for each of them as reported by Avella et al. (2010), were used.

\section{SCFA analysis}

Six feces samples for each rat group (one feces sample for each rat) were analyzed by headspace solid-phase microextraction coupled to gas-chromatography equipped with flame ionization detection as previously reported (Fiorini et al. 2015).

The results were expressed as $\mu \mathrm{mol} / \mathrm{g}$ feces. The precision, as average of the relative standard deviation percentages, 


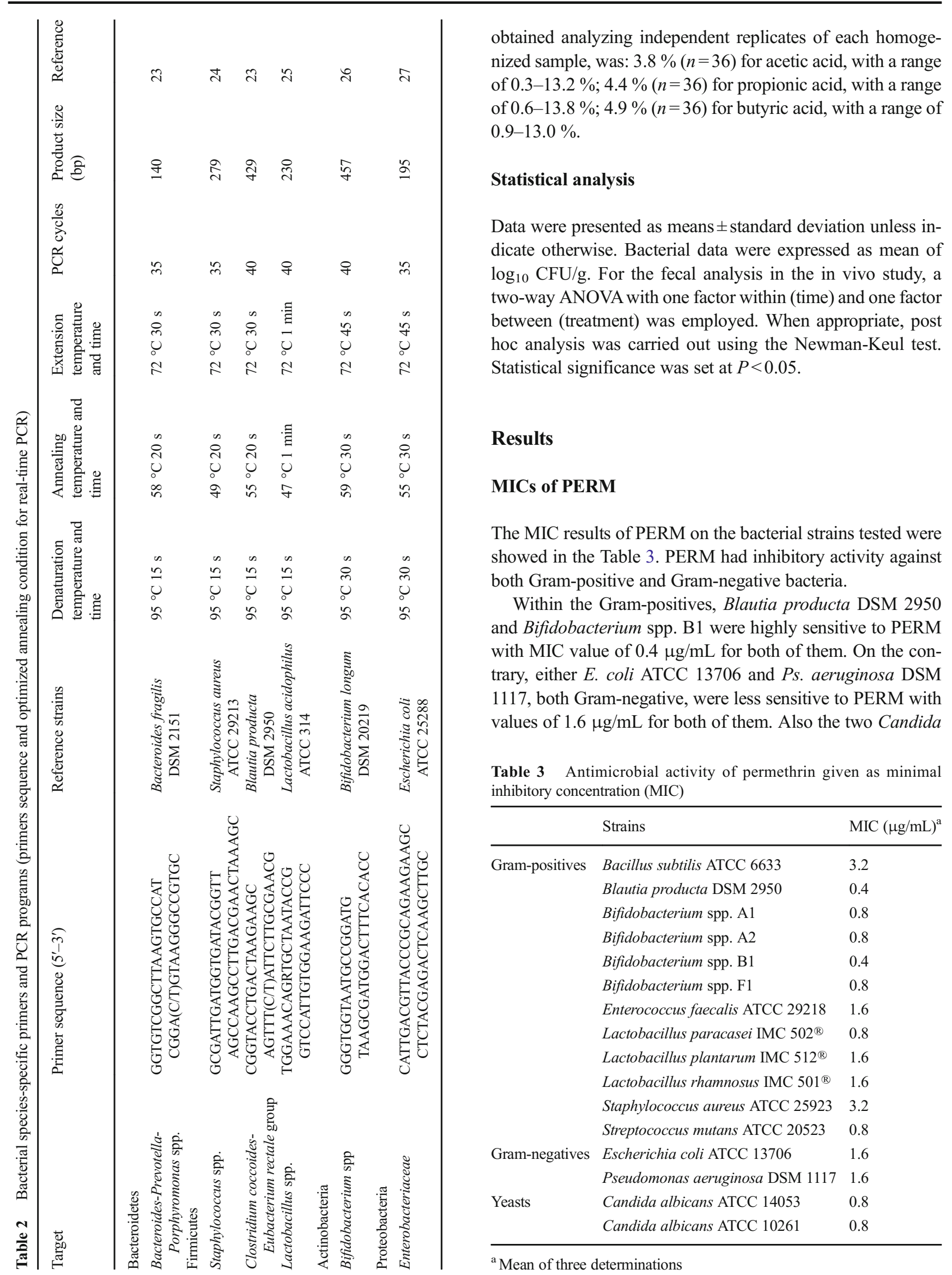


strains were inhibited by values of $0.8 \mu \mathrm{g} / \mathrm{mL}$ of PERM. From the point of view of beneficial and potential pathogen bacteria, PERM was effective with lower concentration on beneficial bacteria such as Bifidobacterium spp. and Lact. paracasei IMC $502^{\circledR}$. While higher concentration was needed to inhibit potential pathogens such as Staphylococcus aureus, Bacillus subtilis, E. coli and Pseudomonas aeruginosa. In the range of pathogens more sensitive were the yeast strains as they required a low amount of this compound to be inhibited.

\section{Effect of PERM on human fecal microflora in a semi-continuous fermentor}

The bacterial counts on the fermentation samples related to all the studied bacterial groups and to all established time points did not show significant modulatory effect of PERM treatment within $96 \mathrm{~h}$ of fermentation in a semi-continuous fermentor mimicking ileotomy fluid when compared with fermentation samples treated with glucose (data not shown).

\section{Effect of PERM on fecal microflora in rats}

The standard curves had a very high efficiency (\%), correlation coefficient $\left(R^{2}\right)$ and slope obtained by plotting the threshold cycle values against the target DNA starting quantity. Using the formula $E=[10(-1 /$ slope $)-1]$, the efficiencies for the individual assays were between 87.0 and $103.4 \%$. All of these parameters indicated a very good quality of the constructed standard curves and so a precise and accurate quantification of the samples. The results from the analysis of rat fecal samples were showed in Table 4.

For Bacteroides-Prevotella-Porphyromonas spp., significant differences between control and PERM-treated groups were observed $(F[1,4]=14.0, P=0.019)$. Post hoc test showed that the PERM-treated group displayed a significant increase in these bacterial species at PND $21(P=0.043)$ and PND $51(P=0.021)$. Conversely, at PND 141, the levels of these species were significantly reduced in PERM-treated rats compared with controls $(P=0.019)$.

For Staphylococcus spp. and Bifidobacterium spp., results revealed no significant difference between control and PERM-treated groups (Staphylococcus spp.: $F[1,4]=0.68$, $P>0.05$; Bifidobacterium spp.: $F[1,4]=1.703, P>0.05)$ at all time points.

For Lactobacillus spp., significant differences between control and PERM-treated groups were observed $(F[1$, $4]=32.82, P=0.004)$. In particular, post hoc test demonstrated that this bacterial species was significantly higher in PERM-treated group compared with that of controls at PND $21(P=0.001)$ and PND $51(P=0.022)$.

For Enterobacteriaceae, ANOVA revealed a significant treatment $\mathrm{x}$ time interaction $(F[3,12]=4.23, P=0.029)$, but not a significant effect of treatment $(F[1,4]=4.54, P=0.10)$. Post hoc analysis revealed that this bacterial family was significantly more abundant in PERM-treated rats than in controls at PND $51(P=0.01)$.

At all time points (PND 21, 51, 81, and 141), daily fecal output and food intake were measured in PERM-treated and control groups. No differences were reported between PERM-

Table 4 Bacterial species quantified using 16S rDNA tags in fecal samples of control and PERM-treated rats over a 4-month follow-up period

\begin{tabular}{|c|c|c|c|c|c|c|}
\hline \multirow[t]{3}{*}{ Bacteria target } & & \multirow[t]{3}{*}{ Group } & \multicolumn{4}{|c|}{ Bacterial concentration $\left(\log _{10} \mathrm{CFU} / \mathrm{g}\right)$} \\
\hline & & & \multirow[b]{2}{*}{ PND 21} & \multicolumn{3}{|c|}{ Treatment follow-up } \\
\hline & & & & PND 51 & PND 81 & PND 141 \\
\hline \multirow{2}{*}{ Bacteroidetes } & \multirow{2}{*}{$\begin{array}{l}\text { Bacteroides-Prevotella- } \\
\text { Porphyromonas spp. }\end{array}$} & Control & $7.29 \pm 0.15$ & $7.17 \pm 0.02$ & $7.19 \pm 0.03$ & $7.00 \pm 0.05$ \\
\hline & & PERM & $7.53 \pm 0.09^{*}$ & $7.58 \pm 0.02 *$ & $7.24 \pm 0.01$ & $6.77 \pm 0.15^{*}$ \\
\hline \multirow[t]{6}{*}{ Firmicutes } & \multirow[t]{2}{*}{ Staphylococcus spp. } & Control & $5.95 \pm 0.07$ & $5.10 \pm 0.02$ & $5.87 \pm 0.08$ & $5.58 \pm 0.18$ \\
\hline & & PERM & $6.14 \pm 0.22$ & $5.91 \pm 0.18$ & $5.74 \pm 0.16$ & $5.30 \pm 0.21$ \\
\hline & \multirow{2}{*}{$\begin{array}{l}\text { Clostridium coccoides- } \\
\text { Eubacterium rectale group }\end{array}$} & Control & Not determined & Not determined & Not determined & $9.73 \pm 0.07$ \\
\hline & & PERM & Not determined & Not determined & Not determined & $9.72 \pm 0.16$ \\
\hline & \multirow[t]{2}{*}{ Lactobacillus spp. } & Control & $3.59 \pm 0.04$ & $3.42 \pm 0.02$ & $3.98 \pm 0.04$ & $3.87 \pm 0.11$ \\
\hline & & PERM & $4.08 \pm 0.07 *$ & $3.85 \pm 0.01 *$ & $4.11 \pm 0.06$ & $3.79 \pm 0.17$ \\
\hline \multirow[t]{2}{*}{ Actinobacteria } & \multirow[t]{2}{*}{ Bifidobacterium spp. } & Control & $4.51 \pm 1.05$ & $4.60 \pm 0.06$ & $5.00 \pm 0.18$ & $3.75 \pm 0.12$ \\
\hline & & PERM & $5.27 \pm 0.54$ & $5.29 \pm 0.12$ & $5.12 \pm 0.17$ & $3.57 \pm 0.19$ \\
\hline \multirow[t]{2}{*}{ Proteobacteria } & \multirow[t]{2}{*}{ Enterobacteriaceae } & Control & $3.99 \pm 0.26$ & $3.23 \pm 0.11$ & $4.41 \pm 0.05$ & $4.10 \pm 0.18$ \\
\hline & & PERM & $4.37 \pm 0.12$ & $3.82 \pm 0.07^{*}$ & $4.34 \pm 0.10$ & $4.14 \pm 0.28$ \\
\hline
\end{tabular}

Data are expressed as mean of $\log _{10} \mathrm{CFU} / \mathrm{g} \pm$ standard deviation

$* P<0.05$ vs control group 
treated and control groups (daily fecal output: $F[1,10]=0.003$, $P=0.95$; daily food intake: $F[1,10]=0.43, P=0.52$ ).

\section{Effect of PERM on fecal SCFA in rats}

Acetic acid, propionic acid and butyric acid levels were reported in Fig. 1.

For acetic acid, two-way ANOVA revealed a significant treatment $\mathrm{x}$ time interaction $(F[3,12]=4.74, P=0.020)$, but not a significant effect of treatment $(F[1,4]=1.94, P>0.05)$ as
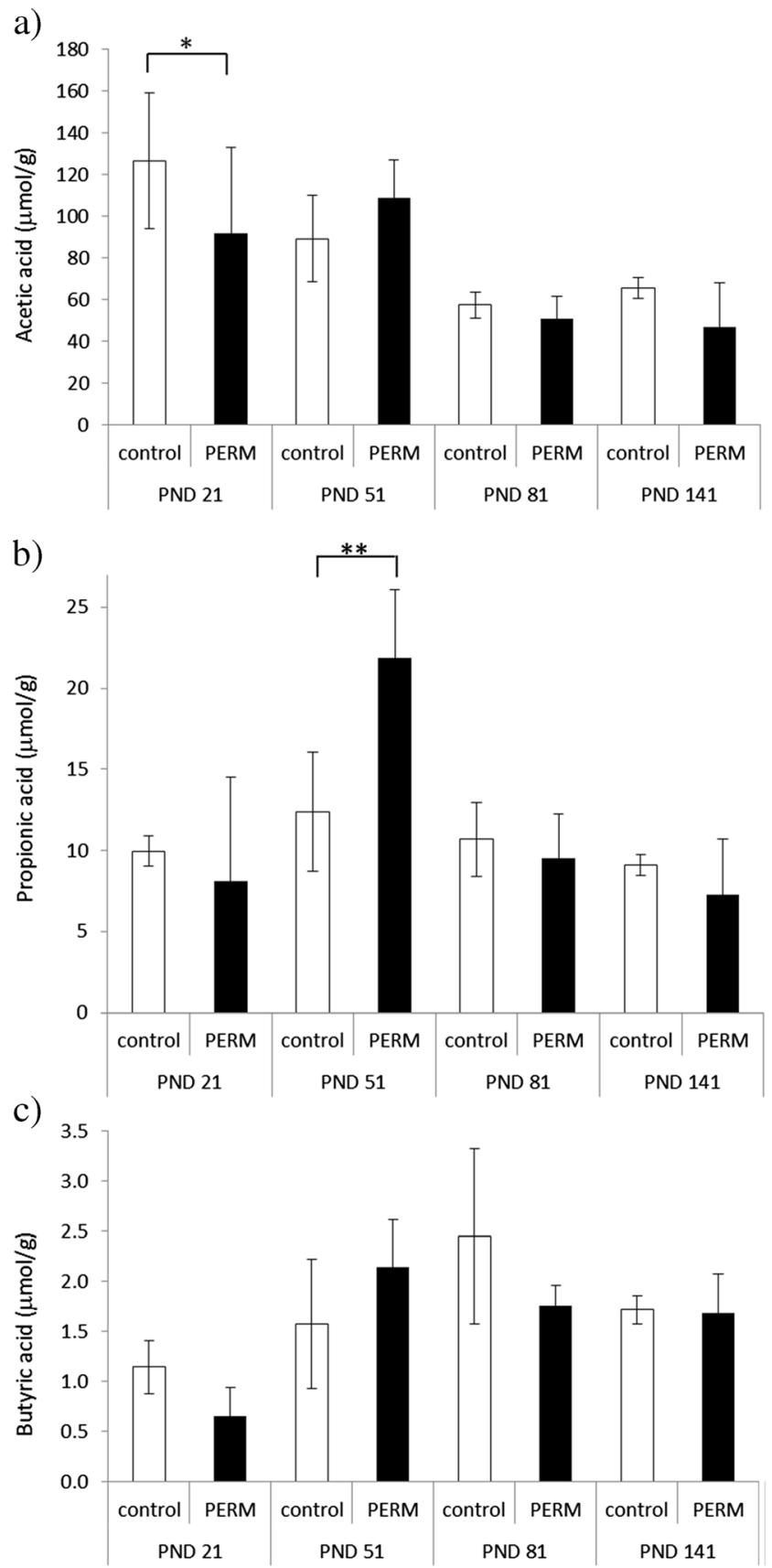

Fig. 1 Quantification of a acetic, b propionic, and $\mathbf{c}$ butyric acids, expressed as $\mu \mathrm{mol} / \mathrm{g}$ feces, in control and PERM-treated rats over a 4month follow-up period. Bars represent means \pm standard deviation shown in Fig. 1a. Post hoc analysis revealed that this SCFA was significantly lower in PERM-treated rats than in controls at PND $21(P=0.017)$.

For propionic acid, two-way ANOVA revealed a significant treatment $\mathrm{x}$ time interaction $(F[3,12]=4.83, P=0.019)$, but not a significant effect of treatment $(F[1,4]=0.004$, $P>0.05$ ) as shown in Fig. 1b. Post hoc analysis revealed that propionic acid was significantly higher in PERM-treated rats than in controls at PND $51(P=0.003)$.

For butyric acid, results revealed no significant treatment $\mathrm{x}$ time interaction $(F[3,12]=3.052, P=0.069)$ and no significant difference between control and PERM-treated groups $(F[1,4]=0.35, P>0.05)$ at all time points (Fig. 1c).

\section{Discussion}

In previous studies, we demonstrated significant effects of permethrin especially on CNS when rat pups were orally exposed to doses $(34 \mathrm{mg} / \mathrm{kg})$ close to the NOAEL for PERM $(25 \mathrm{mg} / \mathrm{kg}$ ) (Carloni et al. 2012; Gabbianelli et al. 2014; Nasuti et al. 2007, 2013, 2014). This pesticide belongs to the pyrethroid family and it is widely used in agriculture, in indoor environments such as households, warehouses, farm and public buildings for pest-control purposes. Consumption of fresh and cooked fruit and vegetables has been linked to higher levels of exposure, as confirmed by the quantification, in human urine, of the 3-phenoxybenzoic acid, the major metabolite of PERM (Barr, et al. 2010). Although the digestive tract is the first organ to come in contact with food contaminants, little is known about the digestive impact of PERM. There are several studies on chlorpyrifos, a pesticide belonging to the organophosphate insecticide, demonstrating how low-dose exposure in perinatal age induces changes in gut microbiota and disrupts gut mucosal barrier (Joly et al. 2013; Joly Condette et al. 2015), but no research on PERM impact on intestinal microbiota has yet been performed.

Since that the population is widely exposed to this pesticide, in this study we attempted to elucidate if early life exposure to PERM could affect gut microbiota monitoring the microbiota evolution over a 4-month follow-up period.

The main finding emerged from this study focused on the reduced abundance of Bacteroides-PrevotellaPorphyromonas species in PERM-treated rats compared to controls at the end of the 4-month follow-up period. In this period of life, the present animal model develops motor disabilities similar of that observed in animal models of Parkinson's disease (paper under submission). The decrease of Prevotella family is associated with dysbiosis of the fecal microbiota in patients with Parkinson's disease (Scheperjans et al. 2015).

Bacteroides species are responsible to produce higher levels of SCFA whose protective role against gut 
inflammation has been well proven (Scheppach et al. 2004). Among them, butyrate plays a particular role for maintaining the intestinal barrier, as shown in inflammatory bowel disease, in which deficit in butyrate causes tight junction lesions and finally impaired intestinal permeability. However, in the present study, no decrease in fecal butyrate was observed in PERM-treated rats versus controls across all times of observation. On the contrary, differences between PERM-treated and control groups were observed for acetic and propionic acids. At the end of the last treatment with PERM (PND 21), acetic acid was decreased in feces of PERM-treated group compared to control. At PND 51, propionic acid was increased in PERM-treated rats. Considering that both groups were fed with the same laboratory diet and no difference was observed in daily food intake and fecal output, the results let us to speculate that PERM treatment could be responsible for the disruption of microbiota composition followed by changes on the end products of fermentation. Particularly, nondigestible carbohydrates are fermented in the proximal colon by primary fermenters like Bacteroides that produce $\mathrm{CO}_{2}$ and lactate. The first is converted to acetic acid by acetogens via the WoodLjungdahl pathway, whereas the second is reduced to propionic acid by other members of the microbial community through the acrylate pathway (Den Besten et al. 2013). Here, in the PERM-treated rats, the bacterial pathway related to acetic acid seems to be partially suppressed whereas the pathway related to propionic acid production appears to dominate over those observed in the control rats. Although diet is a major determinant of gut microbiota, the ingestion of exogenous compound like permethrin may exert a strong influence on the colonic milieu and on the microbial population which in turn affects SCFA production.

Regarding Enterobacteriaceae, which contains most of the opportunistic pathogens, the results showed a trend to a slight increment in their abundance in PERM-treated rats compared to controls at PND 21 followed by a significant increase at PND 51 the second end point. The increase of this bacteria family, especially the pathogenic Escherichia coli strain, is associated with dysbiosis of the fecal microbiota in patients with Parkinson's disease (Scheperjans et al. 2015). Hence, the oral treatment with PERM appears to have changed the normal microenvironment that is suitable for beneficial populations, thereby allowing opportunistic pathogens like Enterobacteriaceae to invade and colonize. However, at PND 81 and PND 141, the Enterobacteriaceae abundance in PERM-treated rats were similar to controls; most probably compensatory mechanisms intervene to mitigate the gut microbiota unbalance observed at the previous time points.
Indeed, the increase of Lactobacillus observed in PERM-treated rats, at PND 21 and PND 51, could represent a compensatory response that, most probably, had prevented the pathogenic Enterobacteriaceae overgrowth at PND 81 and PND 141. Moreover, the findings of in vitro studies ruled out the possibility that PERM in the gut had a trophic effect on lactobacillus because the latter was very sensitive to PERM as shown by a MIC value of $0.8 \mu \mathrm{g} / \mathrm{mL}$.

In the fermentation experiment performed on human fecal microflora, PERM did not have modulatory effects on all bacteria species analyzed. However, Kaufman (1977) found that PERM residues had a short-term inhibitory effect on the functional diversity of soil microbes because microbes can use low-dose PERM as a source of phosphorus or carbon. In the present study, PERM may have a similar impact on bacterial strains. Further, Wagenet (1985) showed that the addition of nutrients to soil may increase the degradation of PERM. Accordingly, we suggest that the addition of low-dose PERM, in these experimental conditions, could be used as an energy source providing a strong benefit to the bacteria species, in fact we observed the same effect when the fermentation was done with addition of glucose.

In conclusion, our data demonstrated that low-dose postnatal exposure to PERM affects negatively the gut microbiota in rats with a decrease in numbers of some strains such as Prevotella that might have a negative impact on gut health. Further studies are warranted to detect the impact of PERM on intestinal mucosa and to include other bacterial families that may increase accuracy, and the potential of fecal microbiome analysis as a biomarker able to detect diseases.

Funding This work was supported by a grant (FAR) from the University of Camerino (Italy).

\section{Compliance with ethical standards}

Conflict of interest The authors report no conflicts of interest relative to the research covered in this manuscript.

\section{References}

Avella MA, Olivotto I, Silvi S, Place AL, Carnevali O (2010) Effect of dietary probiotics on clownfish: a molecular approach to define how lactic acid bacteria modulate development in a marine fish. Am J Physiol Regul Integr Comp Physiol 298:R359-R371

Barr DB, Wong OA, Unubka S, Baker SE, Whitehead RD, Magsumbol MS, Williams BL, Needham LL (2010) Urinary concentration of metabolites of pyrethroid insecticides in the general U.S. population: National Health and Nutrition Examination Survey 1999-2002. Environ Health Perspect 118:742-748 
Bartosch S, Fite A, Macfarlane GT, McMurdo ME (2004) Characterization of bacterial communities in feces from healthy elderly volunteers and hospitalized elderly patients by using real-time PCR and effects of antibiotic treatment on the fecal microbiota. Appl Environ Microbiol 70:3575-3581

Byun R, Nadkarni MA, Chhour KL, Martin FE, Jacques NA, Hunter N (2004) Quantitative analysis of diverse Lactobacillus species present in advanced dental caries. J Clin Microbiol 42:3128-3136

Carloni M, Nasuti C, Fedeli D, Montani M, Amici A, Vadhana MS, Gabbianelli R (2012) The impact of early life permethrin exposure on development of neurodegeneration in adulthood. Exp Gerontol 47:60-66

Casida JE, Durkin KA (2013) Neuroactive insecticides: targets, selectivity, resistance, and secondary effects. Annu Rev Entomol 58:99-117

Den Besten G, van Eunen K, Groen AK, Venema K, Reijngoud DJ, Bakker BM (2013) The role of short-chain fatty acids in the interplay between diet, gut microbiota, and host energy metabolism. J Lipid Res 54(9):2325-2340

Fang H, Hedin G (2003) Rapid screening and identification of methicillin-resistant Staphylococcus aureus from clinical samples by selective-broth and real-time PCR assay. J Clin Microbiol 41: 2894-2899

Fiorini D, Pacetti D, Gabbianelli R, Gabrielli S, Ballini R (2015) A salting out system for improving the efficiency of the headspace solid-phase microextraction of short and medium chain free fatty acids. J Chromatogr A 1409:282-287

Gabbianelli R, Nasuti C, Falcioni G, Cantalamessa F (2004) Lymphocyte DNA damage in rats exposed to pyrethroids: effect of supplementation with Vitamins E and C. Toxicology 203:17-26

Gabbianelli R, Martínez JA, De Caterina R (2014) European Summer School on Nutrigenomics: Camerino, Italy, September 1-5, 2014. J Nutrigenet Nutrigenomics 7:1-19

Heudorf U, Angerer J (2001) Metabolites of pyrethroid insecticides in urine specimens: current exposure in an urban population in Germany. Environ Health Perspect 109:213-217

Joly Condette C, Bach V, Mayeur C, Gay-Quéheillard J, Khorsi-Cauet H (2015) Chlorpyrifos exposure during perinatal period affects intestinal microbiota associated with delay of maturation of digestive tract in rats. J Pediatr Gastroenterol Nutr 61(1):30-40

Joly C, Gay-Quéheillard J, Léké A, Chardon K, Delanaud S, Bach V, Khorsi-Cauet H (2013) Impact of chronic exposure to low doses of chlorpyrifos on the intestinal microbiota in the Simulator of the Human Intestinal Microbial Ecosystem (SHIME) and in the rat. Environ Sci Pollut Res Int 20(5):2726-2734

Kaufman, DD (1977) Permethrin degradation in soil and microbial cultures. In: Elliot Michael (ed) Synthetic Pyrethroids, reprinted from ACS Symposium series No. 42, American Chemical Society, chapter 14, pp 147-161

Clinical and Laboratory Standards Institute (2007) Performance standards for antimicrobial susceptibility testing. Seventeenth informational supplement, CLSI document M100-S17 [ISBN 1-56238-625-5]. Clinical and Laboratory Standards Institute, Wayne, Pennsylvania, 19087-1898 USA.

Langendijk PS, Schut F, Jansen GJ, Raangs GC, Kamphuis GR, Wilkinson MH, Welling GW (1995) Quantitative fluorescence in situ hybridization of Bifidobacterium spp. with genus-specific 16S
rRNA-targeted probes and its application in fecal samples. Appl Environ Microbiol 61:3069-3075

Morelli L (2008) Postnatal development of intestinal microflora as influenced by infant nutrition. J Nutr 138:1791S-1795S

Morgan MK (2012) Children's exposures to pyrethroid insecticides at home: a review of data collected in published exposure measurement studies conducted in the United States. Int J Environ Res Public Health 9(8):2964-2985

Nasuti C, Cantalamessa F, Falcioni G, Gabbianelli R (2003) Different effects of Type I and Type II pyrethroids on erythrocyte plasma membrane properties and enzymatic activity in rats. Toxicology 191(2-3):233-244

Nasuti C, Gabbianelli R, Falcioni ML, Di Stefano A, Sozio P, Cantalamessa F (2007) Dopaminergic system modulation, behavioural changes, and oxidative stress after neonatal administration of pyrethroids. Toxicology 229:194-205

Nasuti C, Falcioni ML, Nwankwo IE, Cantalamessa F, Gabbianelli R (2008) Effect of permethrin plus antioxidants on locomotor activity and striatum in adolescent rats. Toxicology 251:45-50

Nasuti C, Carloni M, Fedeli D, Gabbianelli R, Di Stefano A, Serafina CL, Silva I, Domingues V, Ciccocioppo R (2013) Effects of early life permethrin exposure on spatial working memory and on monoamine levels in different brain areas of pre-senescent rats. Toxicology 303:162-168

Nasuti C, Fattoretti P, Carloni M, Fedeli D, Ubaldi M, Ciccocioppo R, Gabbianelli R (2014) Neonatal exposure to permethrin pesticide causes lifelong fear and spatial learning deficits and alters hippocampal morphology of synapses. J Neurodev Disord 6:7

Rinttila T, Kassinen A, Malinen E, Krogius L, Palva A (2004) Development of an extensive set of 16S rDNA-targeted primers for quantification of pathogenic and indigenous bacteria in faecal samples by real-time PCR. J Appl Microbiol 97:1166-1177

Roberts JR, Karr CJ (2012) Council on Environmental Health: technical report: pesticide exposure in children. Pediatrics 130(6):e1765e1788. doi:10.1542/peds.2012-2758

Scheperjans F, Aho V, Pereira PA, Koskinen K, Paulin L, Pekkonen E, Haapaniemi E, Kaakkola S, Eerola-Rautio J, Pohja M, Kinnunen E, Murros K, Auvinen P (2015) Gut microbiota are related to Parkinson's disease and clinical phenotype. Mov Disord 30(3):350-358

Scheppach W, Weiler F (2004) The butyrate story: Old wine in new bottles? Curr Opin Clin Nutr Metab Care 7:563-567

Silvi S, Rumney CJ, Rowland IR (1996) An assessment of three selective media for bifidobacteria in faeces. J Appl Bacteriol 81:561-564

Wagenet LP (1985) A review of physical-chemical parameters related to the soil and groundwater fate of selected pesticides in N.Y. State. Cornell University, Agricultural Experiment Station, N.Y. State College of Agriculture and Life Sciences, Ithaca, NY, \#30. ISSN 0362-2754

Williams MK, Rundle A, Holmes D, Reyes M, Hoepner LA, Barr DB, Camann DE, Perera FP, Whyatt RM (2008) Changes in pest infestation levels, self-reported pesticide use, and permethrin exposure during pregnancy after the 2000-2001 US Environmental Protection Agency restriction of organophosphates. Environ Health Perspect 116(12):1681-1688

Zampa A, Silvi S, Fabiani R, Morozzi G, Orpianesi C, Cresci A (2004) Effects of different digestible carbohydrates on bile acid metabolism and SCFA production by human gut micro-flora grown in an in vitro semi-continuous culture. Anaerobe 10:19-26 